\title{
Evolução do índice de área foliar de cafeeiro arábica sob diferentes níveis e formas de parcelamentos de adubação
}

\author{
Evolution of index of leaf area of arabic coffee under different levels and forms of manure of \\ installments
}

José Antonio do Vale SantAna ${ }^{l}$

Doutor em Recursos Hídricos em Sistemas Agrícolas Professor no Instituto Federal de Educação, Ciência e Tecnologia Campus Confresa

E-mail: jose.santana@cfs.ifmt.edu.br

Alberto Colombo $^{2}$
Doutor em Engenharia de Irrigação
Professor na Universidade Federal de Lavras).
E-mail: aacolombo@deg.ufla.br
Myriane Stella Scalco ${ }^{3}$
Doutora em Agronomia (Fitotecnia)
Professora na Universidade Federal de Lavras.
E-mail: mscalco@deg.ufla.br
Gleice Aparecida Assis ${ }^{4}$
Doutora em Agronomia (Fitotecnia)
Professor na Universidade Federal de Uberlândia-Campus Monte Carmelo
E-mail: gleiceaufu@gmail.com

Resumo: Este estudo teve como objetivo, avaliar ao longo do tempo, o índice de área foliar do cafeeiro arábica, cultivar Catiguá MG-3, sob diferentes níveis e formas de parcelamentos de adubação. O cafeeiro foi plantado em maio de 2007, no espaçamento de 2,5 x 0,6 metros.O delineamento foi em blocos casualizados, com três repetições, utilizando uma adaptação da análise conjunta de experimentos, com o esquema de parcelas divididas. As duas formas de parcelamentos, quatro (Nov, Dez, Jan e Fev) e 12 (mensais) aplicações, foram consideradas como os ambientes, os níveis de adubação (30\%, 80\%, 130\%, $180 \%$ e $230 \%$ da recomendação de $\mathrm{N}$ e $\mathrm{K} 2 \mathrm{O}$ para cafeeiros cultivados em sequeiros em Minas Gerais), as parcelas e as épocas de avaliação (realizadas bimestralmente entre os anos de 2010 e 2013) constituíram as subparcelas no tempo.Os adubos utilizados foram ureia pecuária $(45 \% \mathrm{de} \mathrm{N}) \mathrm{e}$ nitrato de potássio ( $13 \%$ de $\mathrm{N}$ e $44 \%$ de K2O).Os modelos, Logístico e Gompertz, apresentaram bons ajustes, sendo indicado conforme os avaliadores de qualidade para descrever o índice de área foliar do cafeeiro ao longo do tempo. O índice de área foliar não é influenciado pelas formas de parcelamentos (quatro e doze aplicações/ano). Níveis abaixo de $80 \%$ da adubação com $\mathrm{N}$ e K recomendada para plantas não irrigadas restringe o crescimento do cafeeiro em lavouras irrigadas.

Palavras-chave: Coffe arabicaL.; Fertirrigação; Crescimento.

Abstract: This study aimed to evaluate over time, the leaf area index of Arabica coffee, cultivate Catiguá MG-3 under different levels and forms of fertilizer applications. The coffee was planted in May 2007, in the spacing of 2.5 x 0.6 meters. The design was a randomized 
block design with three replications, using an adaptation of the joint analysis of experiments, with the scheme of split plots. The two forms of installment payments, four (Nov, Dec, Jan and Feb) and 12 (monthly) applications were considered environments, the fertilization levels $(30 \%, 80 \%, 130 \%, 180 \%$ and $230 \%$ of the recommendation $\mathrm{N}$ and $\mathrm{K} 2 \mathrm{O}$ for coffee grown in sequeiros in Minas Gerais), the plots and the evaluation times (held every two months between the years 2010 and 2013) were the subplots in time. The fertilizers were used livestock urea $(45 \% \mathrm{~N})$ and potassium nitrate $(13 \% \mathrm{~N}$ and $44 \% \mathrm{~K} 2 \mathrm{O})$. Models, Logistic and Gompertz, showed good fits and is indicated as quality assessors to describe the leaf area index of the coffee over time. The leaf area index is not influenced by the forms of installment payments (four twelve applications / year). Levels below $80 \%$ of fertilization with $\mathrm{N}$ and $\mathrm{K}$ recommended for non-irrigated plants restricts the growth of coffee in irrigated crops.

Keywords: Coffe arabica L.; fertigation; Growth.

\section{Introdução}

No Brasil, cerca de 251 mil hectares de cafeeiros são irrigados, e o potencial produtivo médio é de 10 milhões de sacas beneficiadas por ano, sendo que 28,6\% das lavouras irrigadas utilizam o sistema de gotejamento, o que corresponde a 71.700 hectares dos 251 mil hectares irrigados (Santinato\& Fernandes, 2012).

De acordo com Fernandes et al. (2007) para que a irrigação seja viável, torna-se necessário adotar práticas que contribuam para o aumento da produtividade e do lucro. Uma destas práticas é a fertirrigação, que tem como benefício a facilidade do parcelamento da adubação em cobertura, fornecendo nutrientes de acordo com a absorção da planta

$\mathrm{Na}$ aplicação via água de irrigação o tempo de chegada dos fertilizantes às raízes das plantas é significativamente reduzido, uma vez que o fertilizante é aplicado juntamente com água. Na adubação convencional, os nutrientes sólidos são depositados próximos da planta e na superfície do solo, ficando dependente da intensidade e frequência das chuvas (Borges \& Coelho, 2009).

Apesar das vantagens que a fertirrigação pode proporcionar com a maximização do aproveitamento dos nutrientes associada a melhor metodologia de aplicação gerando economia de insumos. As recomendações de adubação ainda são pouco específicas para cultura do cafeeiro fertirrigado, não havendo uma unanimidade, quanto aos níveis de adubo que deve ser aplicado.

Rezende et al. (2010) Observaram que houve interação entre regimes hídricos e fertirrigação versus doses de NPK para algumas variáveis de crescimento, porém esses 
resultados não apresentaram tendência comum que permitisse constatar qual a dose mais indicada para cada regime hídrico na fase de crescimento inicial do cafeeiro.

Segundo Sobreira et al. (2011) a adubação de N e K para cafeeiro fertirrigados na formação ( $1^{\circ}$ e $2^{\circ}$ ano pós-plantio) deve ser $30 \%$ inferior à recomendada por Guimarães et al. (1999) para o cultivo em sequeiro. Entretanto Santinato\& Fernandes (2002) recomendam um acréscimo de $30 \%$ no nível de adubação em cafeeiro irrigados em produção.

Em relação ao numérico de aplicações, Coelho et al. (2004) avaliaram diferentes formas de parcelamento da adubação (quatro, doze, vinte e quatro e trinta e seis vezes) por fertirrigação para o cafeeiro arábica e observaram que os números de aplicações não diferiram entre si. Entretanto Sobreira et al., (2011) comparando duas formas de parcelamento (doze e quatro aplicações ao ano), concluíram que no parcelamento de doze vezes há um maior desenvolvimento das plantas, devido a um maior aproveitamento dos nutrientes pela cultura, pois mantém o teor foliar de $\mathrm{N}$ mais adequado durante a sua fase de formação.

Dessa forma, verifica que apesar do manejo da cultura do cafeeiro estar bem consolidado, ainda há espaço para seu aprimoramento na aplicação de fertilizante via água de irrigação. Uma vez que, aplicação excessiva aplicação excessiva de fertilizantes tem um custo social decorrente da contaminação do lenço freático, conforme relata Tumusiime et al. (2011). Para o caso específico de aplicação excessiva de fertilizante, Wang et al. (2014) incluem também nos custos sociais a emissão de gases ligados ao efeito estufa, eutrofização das águas e acidificação do solo.

Assim, o presente estudo teve como objetivo, avaliar ao longo do tempo, o índice de área foliar do cafeeiro arábica, cultivar Catiguá MG-3, sob diferentes níveis e formas de parcelamentos de adubação.

\section{Metodologia}

O experimento foi conduzido no Setor de Cafeicultura do Departamento de Agricultura da Universidade Federal de Lavras (UFLA), no município de Lavras, Minas Gerais. As coordenadas geográficas da referida área são $21^{\circ} 14^{\prime} \mathrm{S}$ de latitude, $44^{\circ} 58^{\prime} \mathrm{W}$ de longitude e altitude média de 910 m. O clima da região é do tipo Cwa, segundo a classificação de Köppen (mesotérmico com verões brandos e suaves e estiagem de inverno) (Dantas et al., 2007). O solo da área experimental é classificado como Latossolo Vermelho distroférrico de textura muito argilosa (Sobreira et al., 2011). 
O plantio do experimento foi realizado em 18 de maio de 2007, no espaçamento de 2,5 $\mathrm{m}$ entre linhas por $0,6 \mathrm{~m}$ entre plantas (6666 plantas ha-1). A cultivar utilizada foi a 'Catiguá MG-3' (Catuaí Amarelo IAC 86 x Híbrido de Timor UFV 440- 10) cujas mudas foram fornecidas pela EPAMIG- Centro Tecnológico do Sul de Minas. A calagem e as adubações de implantação e pós-plantio foram realizadas com base nas recomendações de Guimarães et al. (1999).

As adubações com $\mathrm{N}$ e $\mathrm{K}$ foram feitas via fertirrigação utilizando cinco níveis de adubação em duas formas de parcelamento: quatro vezes ao ano no período das chuvas (Nov., Dez., Jan., Fev.) e doze vezes ao ano, sendo uma aplicação a cada mês, em quantidades iguais. Foram utilizadas como fontes de nutrientes a uréia pecuária, que forneceu parte o $\mathrm{N}$ ( $45 \%$ de $\mathrm{N}$ ) e nitrato de potássio que forneceu $\mathrm{N}$ e $\mathrm{K}\left(13 \%\right.$ de $\mathrm{N}$ e $44 \%$ de $\left.\mathrm{K}_{2} \mathrm{O}\right)$.

$\mathrm{Na}$ fase de crescimento do cafeeiro os cinco níveis de adubação de $\mathrm{N}$ e $\mathrm{K}_{2} \mathrm{O}$ utilizados foram $70 \%, 100 \%, 130 \%, 160 \%$ e $190 \%$ da recomendação para sequeiro de acordo com a recomendação de Guimarães et al. (1999) e na fase de produção esses níveis de adubação estudados foram alterados para 30\%, 80\%, 130\%, $180 \%$ e $230 \%$ da recomendação de sequeiro. Esta mudança foi feita em novembro de 2009, em função da ausência de resposta dos níveis de adubação inicialmente propostos $(70 \%, 100 \%, 130 \%, 160 \%$ e $190 \%$ da recomendação para sequeiro) conforme pode ser constatado em Sobreira et al. (2011), os quais observaram que em ambos os parcelamentos (quatro e doze adubações no ano) o aumento de $90 \%$ ou a redução de $30 \%$ da dose recomendada de $\mathrm{N}$ e $\mathrm{K}_{2} \mathrm{O}$ não alterou o crescimento vegetativo do cafeeiro no primeiro e segundo ano de formação.

O cálculo da adubação foi feito com base na análise foliar do teor de nitrogênio e na análise de solo de cada ano e na produtividade média esperada, segundo a recomendação de sequeiro (Tabela 1$)$.

Tabela 1. Teores médios de K e P encontrados no solo (camada de $0-0,2 \mathrm{~m}$ ), teor foliar e quantidades totais das duas fontes de fertilizantes para $100 \%$ da dose recomendada para o cafeeiro em sequeiro na fase de produção no ano de 2010, 2011, 2012 e 2013.

Análise do solo $\quad$ Teor $\quad$ Fertilizantes totais das duas

\begin{tabular}{|c|c|c|c|c|c|}
\hline \multirow[b]{2}{*}{ Ano } & \multicolumn{2}{|c|}{ Allanse do soio } & $\frac{\text { foliar }}{\mathrm{N}}$ & \multicolumn{2}{|c|}{ fontes } \\
\hline & $\begin{array}{c}\mathrm{P} \\
\mathrm{mg} \mathrm{dm}{ }^{-3}\end{array}$ & $\begin{array}{c}\mathrm{K} \\
\mathrm{mg} \mathrm{dm}\end{array}$ & $\begin{array}{c}\mathrm{N} \\
\text { dag } \mathrm{kg}^{-1}\end{array}$ & $\begin{array}{c}\text { Ureia } \\
\mathrm{kg} \mathrm{ha}^{-1} \mathrm{ano}^{-1}\end{array}$ & $\begin{array}{c}\mathrm{KNO}_{3} \\
\mathrm{~kg} \mathrm{ha}^{-1} \text { ano }^{-1}\end{array}$ \\
\hline 2010 & 87,42 & 76 & 2,8 & 431 & 431 \\
\hline 2011 & 46,3 & 205 & 2,5 & 839 & 171 \\
\hline
\end{tabular}




$\begin{array}{llllll}2012 & 31,03 & 104 & 2,6 & 778 & 772 \\ 2013 & 31,36 & 204 & 2,8 & 934 & 227\end{array}$

O delineamento utilizado no experimento foi o de blocos casualizados, com três repetições. $\mathrm{O}$ experimento foi conduzido em duas áreas adjacentes, uma ao lado da outra, as quais foram diferenciadas pelas duas formas de parcelamento de adubação, em uma área foi utilizada quatro aplicações ao ano (P4) e na outra, doze aplicações (P12) ao ano. Cada uma dessas áreas ocupou $675 \mathrm{~m}^{2}$ com 450 plantas e foi repartida em três blocos (150 plantas por bloco). Dentro de cada bloco foram sorteados os níveis de adubação, 30\%, 80\%, 130\%, 180\% e $230 \%$ da recomendação de $\mathrm{N}$ e $\mathrm{K}_{2} \mathrm{O}$ para cafeeiros cultivados em sequeiro segundo Guimarães et al., (1999). Cada parcela foi composta por dez plantas, sendo as oito centrais consideradas úteis. Para cada linha de tratamento foram deixadas bordaduras duplas, de forma a não haver interferência entre tratamentos.

Utilizou-se o sistema de irrigação por gotejamento espaçado com 2,5 entre linhas laterais e 0,3 entre gotejadores (vazão de $3,75 \mathrm{~L} \mathrm{~h}^{-1}$ ). Em cada linha de plantio foi instalada uma linha lateral, assegurando a formação de uma faixa molhada contínua ao longo das linhas de plantas com 0,6 m de largura. A umidade do solo foi monitorada através do uso de tensiômetros instalados nas profundidades de 0,$10 ; 0,25 ; 0,40$ e $0,60 \mathrm{~m}$, sendo a correspondência entre tensão de água no solo e umidade obtida através das curvas características de umidade do solo, a qual foi previamente determinada em laboratório.

As irrigações foram efetuadas utilizando turnos de rega fixos de duas vezes por semana (terças e sextas-feiras), quando a tensão da água no solo na profundidade de $0,25 \mathrm{~m}$ indicava valores de tensão entre $20 \mathrm{e} 60 \mathrm{kPa}$. Esta faixa de tensão foi adotada para o manejo de irrigação do experimento, por manter o solo com umidade suficiente para o desenvolvimento adequado das plantas, conforme os resultados obtidos por Carvalho et al. (2006) e Assis et al. (2014). Para cálculo da lâmina aplicada, foi considerada a média das leituras dos tensiômetros de 0,10 a $0,60 \mathrm{~m}$ de profundidade.

A partir das medidas de crescimento em altura e diâmetro de copa dos cafeeiros foi avaliado o IAF nos anos de 2010, 2011, 2012 e 2013 para os diferentes níveis e formas de parcelamento utilizado neste estudo.

As medidas de crescimento em altura e diâmetro de copa foram realizadas bimestralmente, totalizando seis épocas de avaliação nos três primeiros anos (Jan, Mar, Mai, Jul, Set e Nov) e três épocas de avaliação no ano de 2013(Jan, Abr e Jun). As medidas de 
altura de planta foram realizadas do colo das plantas até a gema apical dos caules, com auxílio de uma régua graduada e as medidas do diâmetro de copa foram feitas também com auxílio de uma trena, no sentido transversal à linha de plantio.

Com base nos valores de altura média das plantas $(\mathrm{H})$, diâmetro de copa (Dc) e distâncias entre plantas nas linhas de plantio (DP), estimou-se a evolução dos valores de área foliar do dossel (AF em $\mathrm{m}^{2}$ por planta) por meio da Equação 1, que representa a metade da área superficial de um elipsoide:

$\mathrm{AF}=2 \pi\left(\frac{\mathrm{DP}^{\mathrm{P}} \mathrm{DC}^{\mathrm{P}}+\mathrm{DP}^{\mathrm{P}} \mathrm{H}^{\mathrm{P}}+\mathrm{DC}^{\mathrm{P}} \mathrm{H}^{\mathrm{P}}}{3}\right)^{\frac{1}{\mathrm{P}}}$

Em que,

$\mathrm{DP}=$ distância entre planta na linha de plantio (m),

$\mathrm{DC}=$ diâmetro de copa $(\mathrm{m})$,

$\mathrm{H}=$ Altura de planta $(\mathrm{m})$,

$\mathrm{P}=1,6075$.

O IAF foi calculado por meio da relação funcional entre a AF e área disponível às plantas, dada pelo espaçamento $(2,5 \times 0,6 \mathrm{~m})$.

$\mathrm{IAF}=\mathrm{AF} \frac{\mathrm{Np}}{10000}$

Onde,

$\mathrm{AF}=$ área foliar do dossel (AF em $\mathrm{m}^{2}$ por planta),

$\mathrm{Np}=$ número de plantas por hectare.

Devido à importância da comparação entre as duas formas de parcelamento (P4 e P12), os dados de IAF $\left(\mathrm{m}^{2} \mathrm{~m}^{-2}\right)$ de cada época ao longo dos quatro anos $(2010,2011,201 \mathrm{e}$ 2013) de avaliações foram submetidos à análise de variância, utilizando-se para isso, uma adaptação da análise conjunta das duas formas de parcelamentos, conforme Pereira et al. (2014). 
Em seguida, para analise de regressão foram selecionados dois modelos, Logístico (equação 3) e Gompertz (equação 4).

$$
\begin{aligned}
& Y_{L}=a_{L}\left[1+b_{L} e^{\left(-c_{L} t\right)}\right]^{-1} \\
& Y_{G}=a_{G} e^{\left[-b_{G} e^{\left(-c_{G} t\right)}\right]_{(4)}}
\end{aligned}
$$

Sendo que,

$\mathrm{Y}_{\mathrm{G}}=$ valores médios de IAF $\left(\mathrm{m}^{2} \mathrm{~m}^{-2}\right)$ estimados pelo modelo Gompertz;

$\mathrm{Y}_{\mathrm{L}}=$ valores médios de $\mathrm{IAF}\left(\mathrm{m}^{2} \mathrm{~m}^{-2}\right)$ estimados pelo modelo Logístico;

$a_{L}, b_{L}$ e $c_{L}=$ parâmetros de ajuste do modelo Logístico;

$\mathrm{a}_{\mathrm{G}}, \mathrm{b}_{\mathrm{G}}$ e $\mathrm{c}_{\mathrm{G}}=$ parâmetros de ajuste do modelo de Gompertz;

$\mathrm{t}=$ tempo, em dias, transcorrido desde o plantio das mudas no campo (DAP).

Os parâmetros dos modelos foram ajustados aos valores observados por meio da ferramenta Solver do aplicativo Microsoft Excel.

Além do coeficiente de determinação $\left(\mathrm{R}^{2}\right)$, os indicativos estatísticos raiz quadrada da média dos quadrados dos erros (RMSE) e a eficiência do modelo ( $\mathrm{E}_{\mathrm{f}}$ ) foram também utilizados para a avaliação do desempenho dos modelos conforme as equações a seguir:

$$
\mathrm{R}^{2}=1-\frac{\mathrm{SQR}}{\mathrm{SQtot}}
$$

$$
\begin{aligned}
& \text { RMSE }=\sqrt{\frac{1}{n} \sum_{i=1}^{n}\left(O_{i}-E_{i}\right)^{2}} \\
& E_{f}=\frac{\left[\sum_{i=1}^{n}\left(O_{i}-\bar{O}\right)^{2}-\sum_{i=1}^{n}\left(O_{i}-E_{i}\right)^{2}\right]}{\sum_{i=1}^{n}\left(O_{i}-\bar{O}\right)^{2}}
\end{aligned}
$$


em que,

$\mathrm{E}_{\mathrm{f}}=$ eficiência do modelo;

$\mathrm{n}$ = número de dados;

$\mathrm{O}_{\mathrm{i}}=$ valor observado;

$\overline{\mathrm{O}}=$ média do valor estimado; $\mathrm{e}$

$\mathrm{E}_{\mathrm{i}}=$ valor estimado.

Também foi feita a descrição do índice de área foliar (IAF) em função das doses de adubação. Para isso, utilizaram-se os dados obtidos na última avaliação de crescimento (Jun/2013) que foram ajustados ao modelo de Mitcherlich com três parâmetros (equação 8).

$$
Y_{I A F}=a_{j}\left[1-e^{-C_{j}\left(x+b_{j}\right)}\right]
$$

Em que,

$\mathrm{Y}_{\mathrm{IAF}}=$ valores médios de $\mathrm{IAF}\left(\mathrm{m}^{2} \mathrm{~m}^{-2}\right)$ estimados pelo modelo de Mitscherlich;

$a_{j}, b_{j}$ e $c_{j}=$ parâmetros de ajuste do modelo de Mitscherlich.

\section{Resultados e Discussão}

Os resultados referentes à análise de variância são apresentados na Tabela 2. Verificase que todas as fontes de variação, com exceção do parcelamento, dos blocos e da interação bloco*época, apresentaram efeitos significativos pelo teste $\mathrm{F}(\mathrm{P}<0,05)$.

Esse resultado corrobora com os obtidos por Coelho et al. (2004), que avaliaram diferentes parcelamentos da adubação por fertirrigação, adubação convencional, época de irrigação e testemunha sem irrigação para o cafeeiro arábica, e os parcelamentos em quatro, doze, vinte e quatro e trinta e seis vezes não diferiram entre si. Arantes et al. (2006) constataram que o crescimento do cafeeiro foi influenciado pela irrigação, mas não foi pelo parcelamento da fertirrigação após a recepa.

Tabela 2. Análise de variância para o índice de área foliar do cafeeiro, cultivar 'Catiguá MG-3', avaliado em duas formas de parcelamentos (P4 e P12) com diferentes níveis de adubação (30\%, 80\%, 130\%, 180\% e 230\%) ao longo do tempo (entre janeiro de 2010 e abril de 2013).

\begin{tabular}{llllll}
\hline. $\mathrm{FV}$ & GL & SQ & QM & Fc & Pr $>$ Fc \\
\hline
\end{tabular}




\begin{tabular}{lccccl}
\hline Parc & 1 & 0,436390 & 0,436390 & 0,770 & $0,3932^{\mathrm{NS}}$ \\
Bloco(Parc) & 4 & 6,723436 & 1,680859 & 2,966 & $0,0521^{\mathrm{NS}}$ \\
Dose & 4 & 76,377034 & 19,094258 & 33,696 & $0,0000^{*}$ \\
Parc*Dose & 4 & 8,358127 & 2,089532 & 3,687 & $0,0260^{*}$ \\
Erro 1 & 16 & 9,066532 & 0,566658 & & \\
Epoca (ep) & 20 & 512,573812 & 25,628691 & 1058,30 & $0,0000^{*}$ \\
Bloco*ep & 40 & 1,364585 & 0,034115 & 1,409 & $0,0569^{\mathrm{NS}}$ \\
ep*Parc & 20 & 1,814884 & 0,090744 & 3,747 & $0,0000^{*}$ \\
ep*Dose & 80 & 30,593352 & 0,382417 & 15,791 & $0,0000^{*}$ \\
ep*Parc*Dose & 80 & 4,842010 & 0,060525 & 2,499 & $0,0000^{*}$ \\
erro 2 & 380 & 8,718015 & 0,024217 & & \\
\hline \multicolumn{7}{l}{ Total } & 629 & 660,868178 \\
O teste não foi significativo. * Valores significativos pelo teste F, ao nível 5\% de significância. Fonte: Autor
\end{tabular}

Apesar do parcelamento da adubação não ter proporcionado variações no crescimento do cafeeiro, a sua escolha deve ser feita de forma criteriosa, levando em consideração os aspectos do solo, o aproveitamento dos nutrientes pela cultura e a durabilidade do sistema, principalmente no caso do sistema de irrigação localizada por gotejamento.

Verifica-se também por meio da Tabela 2 que a interação tripla foi significativa, assinalando que o índice de área foliar se comporta de forma distinta na presença das duas formas de parcelamentos de adubação sob os diferentes níveis de adubação e épocas de avaliações. Desta forma, fez-se o desdobramento das épocas de avaliações dentro de cada combinação dos parcelamentos com os diferentes níveis de adubação (Tabela 3).

A análise do desdobramento do IAF (Tabela 3) para ajuste do modelo Logístico e Gompertz, apresentou efeitos significativos pelo teste $\mathrm{F}$, ao nível de $1 \%$ de significância ( $\mathrm{p}<$ 0,01) em todas as situações testadas. Assim, foi realizada a descrição matemática entre o índice de área foliar e as épocas de avaliação considerando cada combinação dos parcelamentos com as diferentes doses recomendadas de adubação. As estimativas dos parâmetros dos modelos Logístico e Gompertz, e os indicadores estatísticos RMSE, $\mathrm{E}_{\mathrm{f}}$ e $\mathrm{R}^{2}$ são apresentados na Tabela 4.

Verifica-se que houve um desempenho satisfatório para os modelos utilizados, sendo comprovados pelos valores baixos de RMSE, uma vez que quanto menores os valores absolutos de RMSE melhor o ajuste do modelo. Os altos valores do coeficiente de determinação $\left(\mathrm{R}^{2}\right)$, acima de 0,90 , e a eficiência do modelo igual a 0,9999 , reafirmam o bom desempenho dos modelos Logístico e Gompertz (Tabela 4).

Portanto, os dois modelos considerados apresentaram bons ajustes e segundo os avaliadores de qualidade podem ser aplicados para descrever o índice de área foliar do 
cafeeiro ao longo do tempo. Esses resultados estão de acordo com aqueles encontrados por Pires \&Calegario (2007) que ao comparar os modelos lineares e não lineares na descrição do crescimento de plantas, concluíram que o Logístico foi o melhor modelo não-linear e o Gompertz pode ser utilizado com segurança, sendo superior ao modelo de Weibull.

Pereira et al. (2014) avaliaram as variáveis altura de planta por meio dos modelos não lineares ( Logístico e Gompertz), ambos em função das diferentes densidades de plantio, 3333 e 10000 plantas ha $^{-1}$, e regimes de irrigação(sem irrigação, $20 \mathrm{kPa}$ e $60 \mathrm{kPa}$ ) ao longo do tempo e concluíram que o modelo Gompertz é o que melhor representa o crescimento em altura de plantas do cafeeiro

Tabela 3. Análise de variância referente ao desdobramento das épocas de avaliação dentro de cada combinação das formas de parcelamentos (P4 e P12) com os diferentes níveis de adubação $(30 \%, 80 \%, 130 \%$, $180 \%$ e $230 \%$ ), apresentando os números de graus de liberdade (GL), os valores dos quadrados médios (QM) para o índice de área foliar do cafeeiro, cultivar 'Catiguá MG-3', considerando os ajustes do modelo Logístico e Gompertz.

\begin{tabular}{lccc}
\hline & & Logístico & Gompertz \\
FV & GL & QM & QM \\
\hline (Epoca/ P4 e 30\%) & 20 & $0,9253^{* *}$ & $0,9253^{* *}$ \\
Regressão & 3 & $5,7338^{* *}$ & $5,7128^{* *}$ \\
Desvio & 17 & $0,07676^{* *}$ & $0,0805^{* *}$ \\
(Epoca/ P4 e 80\%) & 20 & $2,0854^{* *}$ & $2,0854^{* *}$ \\
Regressão & 3 & $13,7212^{* *}$ & $13,7133^{* *}$ \\
Desvio & 17 & $0,03198^{\mathrm{NS}}$ & $0,0334^{\mathrm{NS}}$ \\
(Epoca/ P4 e 130\%) & 20 & $2,8172^{* *}$ & $2,8172^{* *}$ \\
Regressão & 3 & $18,4515^{* *}$ & $18,4706^{* *}$ \\
Desvio & 17 & $0,0581^{* *}$ & $0,0548^{* *}$ \\
(Epoca/ P4 e 180\%) & 20 & $4,4341^{* *}$ & $4,4341^{* *}$ \\
Regressão & 3 & $29,1398^{* *}$ & $29,1548^{* *}$ \\
Desvio & 17 & $0,0743^{* *}$ & $0,0716^{* *}$ \\
(Epoca/ P4 e 230\%) & 20 & $3,2393^{* *}$ & $3,2393^{* *}$ \\
Regressão & 3 & $21,1816^{* *}$ & $21,1621^{* *}$ \\
Desvio & 17 & $0,0730^{* *}$ & $0,0765^{* *}$ \\
(Epoca/ P12 e 30\%) & 20 & $0,9799^{* *}$ & $0,9799^{* *}$ \\
Regressão & 3 & $6,1013^{* *}$ & $5,9299^{* *}$ \\
Desvio & 17 & $0,0671^{* *}$ & $0,1064^{* *}$ \\
(Epoca/ P 12 e 80\%) & 20 & $2,0386^{* *}$ & $2,0386^{* *}$ \\
Regressão & 3 & $13,3867^{* *}$ & $13,3789^{* *}$ \\
Desvio & 17 & $0,0359^{\mathrm{NS}}$ & $0,0373^{\mathrm{NS}}$ \\
(Epoca/ P 12 e 130\%) & 20 & $3,7523^{* *}$ & $3,7523^{* *}$ \\
Regressão & 3 & $24,3725^{* *}$ & $24,4581^{* *}$ \\
& & &
\end{tabular}


Desvio

(Epoca/ P 12 e 180\%)

Regressão

Desvio

(Epoca/ P 12 e 230\%)

Regressão

Desvio
17

20

3

17

20

3

17
$0,1134 * *$

$3,1245^{* *}$

$24,3725^{* *} *$

$0,1171^{* *}$

$4,0946 * *$

$26,7827 * *$

$0,0909 * *$
$0,0983 * *$

$3,1245 * *$

$20,0598 * *$

$0,1359 * *$

$4,0946 * *$

$26,7150 * *$

$0,1028 * *$

${ }^{\mathrm{NS}} \mathrm{O}$ teste não foi significativo. ** Valores significativos pelo teste $\mathrm{F}$, ao nível 1\% de significância. Fonte: Autor.

Tabela 4. Parâmetros dos modelos Logístico e Gompertz, e os indicadores, RMSE, E e R ${ }^{2}$, para as duas formas de parcelamentos (P4 e P12) com os diferentes níveis de adubação (30\%, 80\%, 130\%, 180\% e 230\%) definidos a partir dos dados observado ao longo do tempo (entre janeiro de 2010 e abril de 2013).

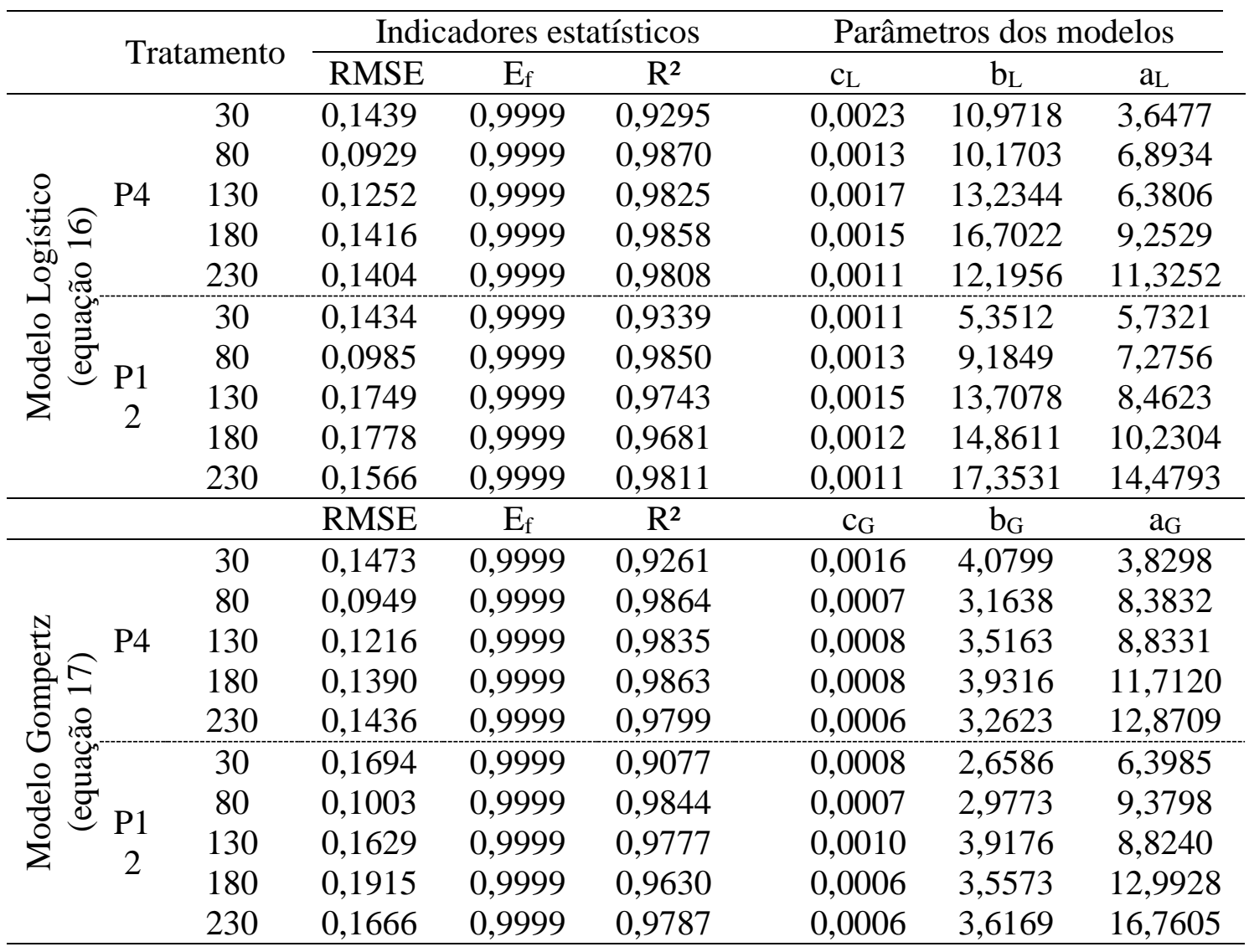

Fonte: Autor. 
Tanto o modelo de Gompertz quanto o modelo Logístico apresentaram valores coerentes para as estimativas dos parâmetros das equações que descreve o crescimento dos cafeeiros, cultivar 'Catiguá MG-3'. Observa-se que os valores obtidos para o índice de maturidade, parâmetro $\left(c_{L} e c_{G}\right)$, foram bem próximos, apresentando uma pequena diferença $(\geq 0,006)$ entre os níveis de adubações utilizadas para a forma de parcelamento em 12 aplicações ao ano. $\mathrm{Na}$ forma de parcelamento com quatro aplicações ao ano, também foi constata essa pequena diferença $(\geq 0,006)$ entre os níveis de $80 \%, 130 \%, 180 \%$ e $230 \%$ (Tabela 4).

Em relação aosparâmetros, aL e aG, nota-se que houve uma resposta crescente em função do aumento nas doses de adubação independente das formas de parcelamento (Tabela $4)$.

A partir dos resultados constata-se também que o manejo de adubação com doze aplicações proporciona incrementos mais acentuados no índice de área foliar, conforme pode ser observado por meio dos parâmetrosa $a_{\mathrm{L}} \mathrm{a}_{\mathrm{G}}$, que sempre atingiu valores maiores neste manejo, indicando que quando bem nutrindo sem restrição hídrica, a planta de café pode apresentar incrementos significativo durante todo o ano.

Também foi feita a descrição do índice de área foliar (IAF) em função das doses de adubação (Figura 1). O modelo Mitcherlich foi escolhido por ser mais adequado, uma vez que a Lei de Mitcherlich ou dos incrementos decrescentes, delineia que apesar do aumento na variável resposta, com o incremento do nível de adubação, esse aumento não acontece de forma proporcional, e isso foi observado na resposta do Índice de área foliar (IAF) em função dos níveis de $\mathrm{N}_{\text {e }} \mathrm{K}_{2} \mathrm{O}$.

O acréscimo no IAF foi de 2,23 (30 e 230\%), 1,35 (80 e 230\%), 0,79 (130 e 230\%) e $0,71 \mathrm{~m}^{2} \mathrm{~m}^{-2}(180$ e $230 \%)$ para o manejo de adubação com doze aplicações ao ano e 2,23 (30 e $230 \%), 1,09$ (80 e $230 \%$ ) e 0,61 (130 e 230\%) para o manejo de adubação com quatro aplicações, que apresentou decréscimo de $-0,71 \mathrm{~m}^{2} \mathrm{~m}^{-2}$ entre $180 \%$ e $230 \%$ (Figura 1).

Figura 1.Medias dos valores de índice de área foliar $\left(\mathrm{m}^{2} \mathrm{~m}^{-2}\right)$ da ultima avaliação de crescimento (Jun/2013) para cafeeiros fertirrigados em duas formas de parcelamentos de adução (P4 e P12), sob diferentes níveis de adubação $(30,80,130,180$ e $230 \%)$. 


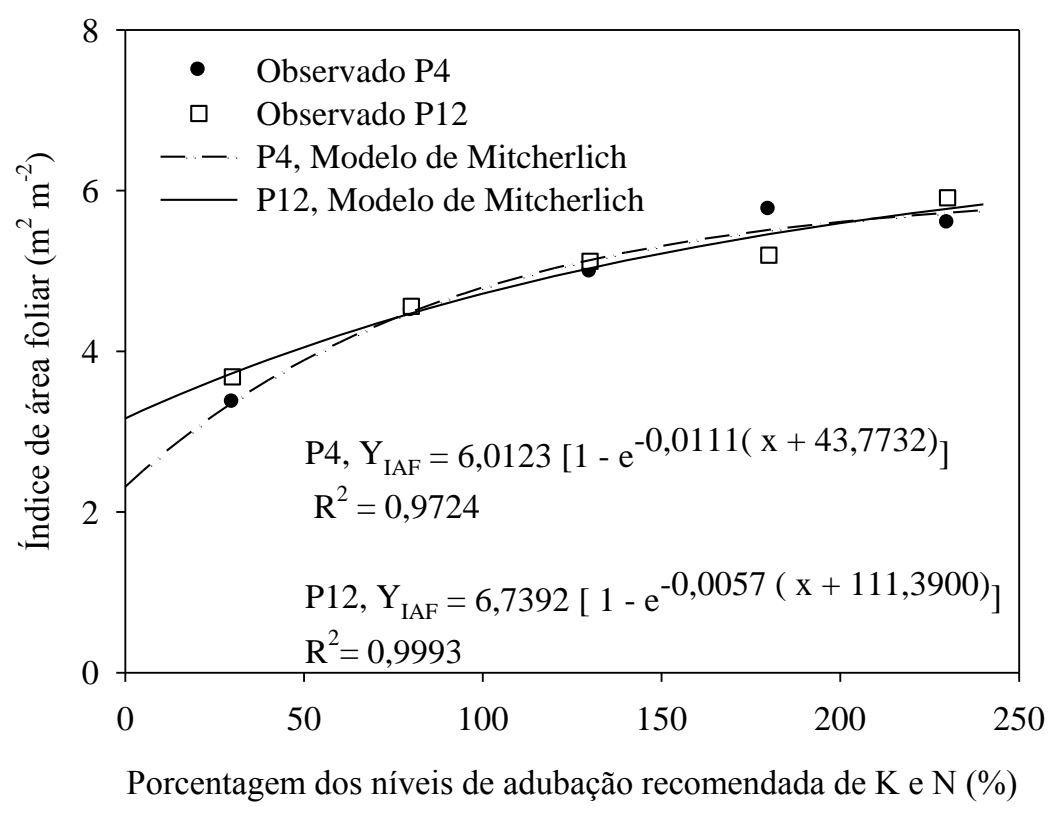

Fonte: Autor.

Os menores níveis de aplicação de $\mathrm{N}$ e $\mathrm{K}_{2} \mathrm{O}$ (30\% e 80\%) foram insuficientes para promover o mesmo padrão de crescimento das plantas em relação às demais doses utilizadas, porém a aplicação de doses entre 130 e $230 \%$ não promoveram diferenças expressivas no Índice de área foliar $\left(<0,8 \mathrm{~m}^{2} \mathrm{~m}^{-2}\right)$ dos cafeeiros fertirrigados em quatro e doze parcelamentos (Figura 1). Esses resultados estão de acordo com a recomendação de Santinato e Fernandes (2002), que sugerem um acréscimo de 30\% na dose de adubação em lavouras irrigadas de cafeeiro em produção.

Níveis de adubações acima de $100 \%$ do recomendado para sequeiro de acordo com a recomendação de Guimarães et al. (1999) também foram encontrados por Costa et al. (2010) que obtiveram valor de $154,17 \%$.

Sobreira et al., (2011) não encontraram diferenças significativas no crescimento de plantas fertirrigadas em aplicações de níveis de adubação variando entre 70 e $190 \%$ para cafeeiros em fase de formação, o que significou uma economia de $30 \%$ com N e $\mathrm{K}_{2} \mathrm{O}$ e maior eficiência com o uso da fertirrigação. No entanto, Carelli et al. (1989), relataram que cafeeiros em fase de frutificação apresentaram maior absorção de nutrientes, assim como maior taxa de fotossíntese, sugerindo que, na presença de frutos, as plantas tornam-se metabolicamente mais ativas.

$\mathrm{Na}$ lavoura em produção a demanda nutricional pode ser maior, pois parte dos nutrientes das folhas é redistribuída para os frutos e, portanto, aumento nos níveis de adubação pode ser indicado como forma de aumentar a produtividade sem prejuízo ao 
crescimento. Neste caso a eficiência da fertirrigação pode estar relacionada ao aproveitamento de maiores doses de $\mathrm{N}$ e $\mathrm{K}_{2} \mathrm{O}$ e não a economia de aplicação como verificado na fase de formação.

\section{Considerações Finais}

Os modelos, Logístico e Gompertz, apresentaram bons ajustes, sendo indicado conforme os avaliadores de qualidade para descrever o índice de área foliar do cafeeiro ao longo do tempo.

O índice de área foliar não é influenciado pelas formas de parcelamentos (quatro e doze aplicações/ano).

Níveis abaixo de $80 \%$ da adubação com N e K recomendada para plantas não irrigadas restringe o crescimento do cafeeiro em lavouras irrigadas.

Níveis de adubações acima do patamar de $130 \%$ não promovem diferenças expressivas no índice de área foliar $\left(<0,8 \mathrm{~m}^{2} \mathrm{~m}-2\right)$ das plantas de cafeeiros fertirrigadas em quatro e doze parcelamentos.

\section{Referências}

Arantes, K. R.; Arantes, S. A. C. M.; Faria, M. A \& Rezend, F. C. (2006). Desenvolvimento do cafeeiro(coffeaarabica L) podado sob irrigação. Revista de Ciências Agro-Ambientais, Alta Floresta, 4, 75-86.

Assis, G. A.; Guimarães, R. J.; Scalco, M. S.; Colombo, A.; Morais, A. R. \& Carvalho, J. P. S (2014). Correlação entre crescimento e produtividade do cafeeiro em função do regime hídrico e densidade de plantio. BioscienceJournal, Uberlândia, 30, 666-676.

Borges, A. L. \&Coelho, E. F. (2009). Fertirrigação em fruteiras tropicais. Cruz das Almas: EMBRAPA Mandioca e Fruticultura Tropical, $2^{\mathrm{a}}$ edição, 180 p.

Carelli, M. L. C.; Fahl, J. I. \&Magalhães, A. C. (1989). Assimilação de nitrato durante o desenvolvimento reprodutivo de plantas de café. Revista Brasileira de Ciência do Solo, Campinas, 13, 59-64.

Carvalho, C. H. M.; Colombo, A.; Scalco, M. S. \& Morais, A. R. (2006).Evolução do crescimento do cafeeiro (Coffea arábica L.) irrigado e não irrigado em duas intensidades de plantio. Ciência eAgrotecnologia, Lavras, 30, 243-250.

Coelho, G.; Silva, A.M.; Silva, R.A.; Oliveira, P.; Silva, A.C.; Sato, F.A. \&Lago, F.J. (2004). Épocas de irrigação e parcelamento da adubação sobre a produtividade do cafeeiro arábica com 17 anos de idade. In: Irriga, 9, 12-18. 
COSTA, A. R.; Rezende, R.; Freitas, P. S. L.de; Frizzone, J. A. \&Helbel Júnior, C. (2010). Número de ramos plagiotrópicos e produtividade de duas cultivares de cafeeiro utilizando irrigação por gotejamento. Revista CiênciaAgronômica, 41, 571-581.

Dantas, A. A. A.; Carvalho, L. G. \&Ferreira, E. (2007). Classificação e tendências climáticas em Lavras. Ciência e Agrotecnologia, Lavras, 31, 1862-1866.

Fernandes, A. L. T.; Santinato, R.;Drumond, L. C. D. \&Oliveira, C. B. de. (2007).Avaliação do uso de fertilizantes organominerais e químicos na fertirrigação do cafeeiro irrigado por gotejamento. Revista Brasileira de Engenharia Agrícola e Ambiental. 11, 159-166.

Guimarães, P. T. G.; Garcia, A. W. R.; Alvarez, V. H.; Prezotti, L. C.; Viana, A. S.; Miguel, A. E.; Malavolta, E.; Corrêa, J. B.; Lopes, A. S.; Nogueira, F. D. \&Monteiro, A. V. C. Cafeeiro. In: Ribeiro, A. C.; Guimarães, P. T. G. \&Alvares, V. H. (Ed). (1999)Recomendação para o uso de corretivos e fertilizantes em Minas Gerais: $5^{\text {a }}$ aproximação.Viçosa, MG: UFV, p.289-302.

Pereira, A. A.; Morais, A. R.; Scalco, M. S. \&Fernandes, T. J. (2014). Evolução do crescimento do cafeeiro (Coffeaarabica L.) irrigado e não irrigado em duas intensidades de plantio. Coffee Science, Lavras, 9, 266-274.

Pires, L. M. \&Calegario, N. (2007). Ajuste de modelos estocásticos lineares e não-lineares para a descrição do perfil longitudinal de árvores. Revista Árvore [online]. 31, 845-852. ISSN 0100-6762. http://dx.doi.org/10.1590/S 0100-67622007000500008.

Rezende, R.; Helbel Júnior, C.; Souza, R. S. de; Antunes, F. M. \&Frizzone, J. A. (2010). Crescimento inicial de duas cultivares de cafeeiro em diferentes regimes hídricos e dosagens de fertirrigação. Engenharia Agrícola, 30, 447-458.

Santinato, R. \&Fernandes, A. L. T. (2002). Cultivo do cafeeiro irrigado em plantio circular sob pivô central. 1ed. Belo Horizonte: O lutador, 251p.

Santinato, R. \& Fernandes, A. L. T. (2012). Cultivo do cafeeiro irrigado por gotejamento. 2. ed. Uberaba: PLD, 388 p.

Sobreira, F. M.; Guimarães, R. J.; Colombo, A.; Scalco, M. S. \&Carvalho, J. G. (2011).Adubação nitrogenada e potássica de cafeeiro fertirrigadona fase de formação, em plantio adensado. Pesquisa agropecuária brasileira, Brasília, 46, 9-16.

Tumusiime, E.; Brorsen, B.W.; Mosali, J. \&Biermacher, J.T. (2011). ' How Much Does Considering the Cost of Lime Affect the Recommended Level of Nitrogen?', Agronomy Journal, 103, 404-412.

Wang, G. L,; Ye,Y. L.; Chen, X. P. \&Cui, Z. L. (2014). Determining the optimal nitrogen rate for summer maize in China by integrating agronomic, economic, and environmental aspects. Biogeosciences Discussions, 11, 2639-2664. 